\title{
Influence of ionic liquids on the dielectric relaxation behavior of CNT based elastomer nanocomposites
}

\author{
D. Steinhauser ${ }^{1}$, K. Subramaniam ${ }^{2}$, A. Das $^{3}$, G. Heinrich $^{2,3}$, M. Klüppel $^{*}$ \\ ${ }^{1}$ Deutsches Institut für Kautschuktechnologie e.V., Eupener Str. 33, 30519 Hannover, Germany \\ ${ }^{2}$ Leibniz-Institut für Polymerforschung Dresden e.V. , Hohe Str. 6, 01069 Dresden, Germany \\ ${ }^{3}$ Technische Universität Dresden, Institut für Werkstoffwissenschaft Helmholtzstrasse 7, 01069 Dresden, Germany
}

Received 2 April 2012; accepted in revised form 24 June 2012

\begin{abstract}
The influence of an imidazolium type ionic liquid (IL) on the relaxation behavior of carbon-nanotube (CNT) based polychloroprene nanocomposites prepared by melt mixing has been investigated by broadband dielectric spectroscopy. It is demonstrated that the presence of the ionic liquid modifies the relaxation behavior of the pure rubber matrix and leads to a significant increase of the conductivity for the CNT/rubber composites. For the unfilled rubber, a distinct glass transition of the IL is observed for high concentrations demonstrating that the IL forms a separate phase. The increased conductivity of the CNT-filled rubber composites is related to a physical coupling between CNTs and rubber matrix mediated by IL leading to a better dispersion of the CNTs.
\end{abstract}

Keywords: nanocomposites, rubber, ionic liquid, dielectric spectroscopy, relaxation dynamics

\section{Introduction}

Electrically conducting rubber has attracted considerable attention due to the potential opportunity of combining the stretchable and deformable properties of the material with sufficient high electric conduction for various applications such as electromagnetic interference shielding materials, sensors and to avoid electrostatic charging. For example, a conductivity of $1 \mathrm{~S} / \mathrm{cm}$ is typically sufficient for electromechanical interference shielding [1]. In technical rubber goods the low electrical conductivity of the polymer is improved by the incorporation of conductive fillers like carbon black. Polymer nano-composites can have a remarkable piezoresistivity $[2,3]$. Such materials are good candidates to be used as sensors with high sensitivity [4]. For example, a piezo-resistive rubber sensor vulcanized into the sidewall of a tire can monitor the actual state of a tire such as the tire pressure to a control system. This kind of pressure control could be realized by measuring the variation of resistivity when the sensor passes through the contact area of a rolling tire reflecting the bending state of the side wall. Such facilities would improve the security and drive stability of a vehicle, significantly.

For a sufficient high conductivity the building up of a filler network is necessary. This can be achieved above the percolation threshold describing the amount of conductive filler necessary to obtain an infinite cluster, which forms a conductive path through the whole sample. Depending on the structure and the size of conductive carbon black particles and the specific interaction with the used polymer, percolation thresholds in the range of $8 \mathrm{vol} \%<$ $\Phi_{\mathrm{c}}<20 \mathrm{vol} \%$ of carbon black are found $[5,6]$. For special conductive carbon blacks the threshold can even go down to 3 vol\% [7]. Recently, also single walled CNT and multi walled carbon-nanotubes 
(MWCNT) have been applied as conductive fillers for reaching the necessary conduction level [8-11]. Compared to carbon black, the percolation threshold of MWCNT is significantly lower due to the high aspect ratio of the tubes. It can be reduced theoretically down to $\Phi_{\mathrm{c}}=0.1 \mathrm{vol} \%$, which can be achieved in case of optimal dispersion resulting in bon black composites [12]. In particular, the very small amount of CNTs necessary to reach high conductivities is an ideal candidate to be used for preparing highly conducting materials with nonconductive reinforcing fillers. Additionally, the use of non-conductive fillers like silica can improve the dispersion of the CNTs due to the increased shearing forces. It has been demonstrated that the incorporation of CNT in silica filled rubber results in a high shear forces during the mixing process [13]. The materials show an enhanced mechanical stiffness and tensile strength, an increased modulus and a high electrical conductivity with quite low amounts of CNT $\left(\Phi_{\mathrm{c}}=1 \mathrm{vol} \%\right)$, though the tear resistance under dynamical loading is slightly reduced. The dynamic-mechanical and dielectric spectra have been analyzed to get more insight into the morphology and dynamics of the systems. Thereby, a better understanding of the conduction mechanism, the polymer-tube interaction and the filler networking in CNT-nanocomposites has been achieved.

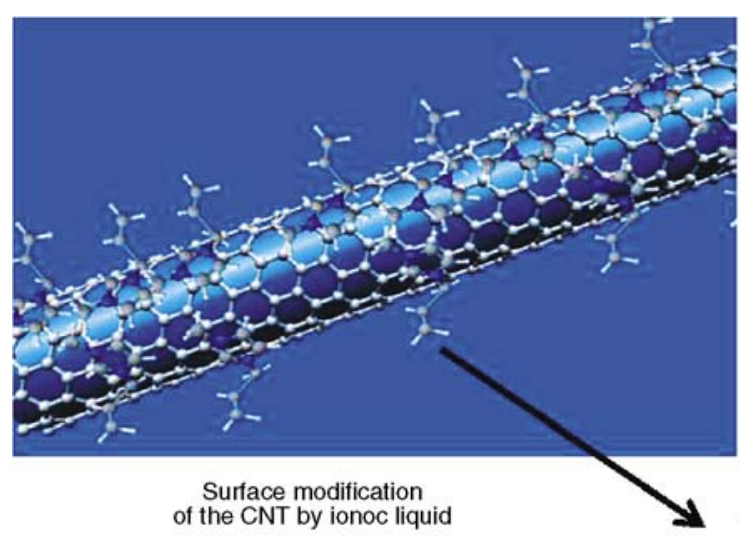
even higher conductivities as for highly filled cargood dispersion of the CNT probably due to the

In previous papers, the conductivity of MWCNT/ rubber composites was found to be increased by applying imidazolium type ionic liquids as dispersion agent $[10,13,14]$. A chemical coupling between CNTs and the rubber matrix mediated by imidazolium type ionic liquids and sulfur bridges can be achieved in SSBR-BR/CNT systems [14] as depicted in Figure 1. For polychloroprene rubber (CR), the usage of 1-butyl 3-methyl imidazolium bis-(trifluoromethyl-sulphonyl)-imide (BMI) modified tubes leads to a physical coupling between CNTs and rubber evidenced by Raman spectroscopy, where the ion-dipole interaction persists probably between $\mathrm{CR}$ and $\mathrm{BMI}$ $[14,15]$. A low amount of MWCNT in polychloroprene rubber showed a very high conductivity level of about $0.1 \mathrm{~S} / \mathrm{cm}$ with a high stretchability of more than 500\%. Transmission electron microscopy (TEM) demonstrated an improved dispersion of the BMI modified tubes in the rubber matrix.

In the present paper, we will extend these investigations by a detailed study of the dielectric properties of the IL-modified MWCNT/rubber composites. We will first investigate the modified relaxation behavior of the pure rubber matrix. In a second step we will consider the dielectric spectra of the MWCNT-filled samples more closely.

\section{Experimental and methods}

The samples were based on chloroprene rubber (CR, Baypren 611, Lanxess AG, Leverkusen, Ger-
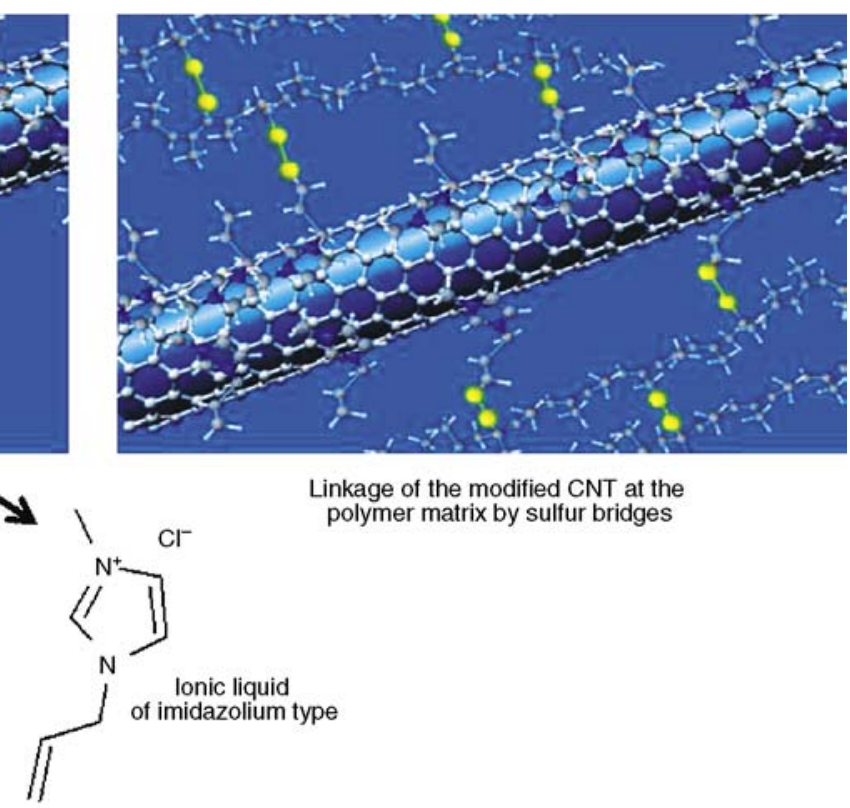

Figure 1. Schematic representation of the coupling between CNT and the rubber matrix mediated by imidazolium type ionic liquids and sulfur bridges 
many). Each composite contained $0.5 \mathrm{phr}$ stearic acid, $5 \mathrm{phr}$ zinc oxide ( $\mathrm{ZnO}), 4 \mathrm{phr}$ magnesium oxide $(\mathrm{MgO})$ and $1 \mathrm{phr}$ ethylene thiourea. These chemicals are used on industrial grades. Table 1 shows the curing recipe and explanation for sample designation. For the CNT/CR composites, the commercial multiwalled carbon nanotubes (MWCNTs) Nanocyl 7000 (Nanocyl S.A, Sambreville, Belgium) has been used. The MWCNTs were modified with the IL, BMI (1-butyl 3-methyl imidazolium bis(trifluoromethylsulphonyl) imide, Sigma-Aldrich, Steinheim, Germany), by grinding them till a black paste was obtained. The MWCNTs were mixed with CR in a laboratory scale two roll mixing mill (Polymix 110L, size: $203 \times 102 \mathrm{~mm}$, Servitech GmbH, Wustermark, Germany) at $40^{\circ} \mathrm{C}$ with a friction ratio of $1: 1.25$. The vulcanization additives were incorporated into the matrix after the admixing of MWCNTs. The optimum cure time $\left(t_{90}\right)$ was determined in a moving die rheometer, shortly referred as MDR (Scarabaeus V50, Scarabaeus GmbH, Langgöns, Germany) at $160^{\circ} \mathrm{C}$ for 60 minutes. The mixed stocks were cured in a standard hot press at $160^{\circ} \mathrm{C}$, $150 \mathrm{kN}$ pressure and at an optimum cure time $\left(t_{90}\right)$ of the samples.

Dielectric investigations have been carried out by using a broadband dielectric spectrometer BDS 40 (Novocontrol GmbH, Hundsangen, Germany). The temperature was varied from -100 to $150^{\circ} \mathrm{C}$ in steps of five degrees using the temperature control system Novocool (Novocontrol GmbH, Hundsangen, Germany). At each temperature, a frequency sweep from $0.1 \mathrm{~Hz}$ to $10 \mathrm{MHz}$ has been measured. The sample geometry was a disc shaped plate capacitor with a diameter of around $2 \mathrm{~cm}$. The sample with a thickness of around $2 \mathrm{~mm}$ was placed between two gold-plated electrodes. To provide an excellent contact between sample and electrodes, thin gold layers have been sputtered onto the flat surface of the sample plate. The dielectric permittivity and the dielectric loss have been recorded for each sample in dependence on temperature and frequency.

\section{Results and discussion}

\subsection{Relaxation dynamics in neat chloroprene rubber}

The dielectric loss $\varepsilon^{\prime \prime}$ of neat CR rubber in dependence on temperature and frequency is shown in Figure $2 \mathrm{a}$. The pronounced relaxation process can be related to the glass transition ( $\alpha$ process) due to the cooperative segmental motion of the chain, for which the characteristic temperature and frequency dependence is described by a Vogel-Fulcher behavior. At low temperature, the Arrhenius-activated $\beta$ process can be observed which is due to local fluctuations of chain segments or side groups. The dielectric loss for CR rubber with IL, shown in Figure 2b, has an additional relaxation process below the glass transition of the rubber, which can therefore be associated with the ionic liquid. It has been reported that ionic liquids, which exhibit disordered and fragile structure, can also form a glassy-like state that could be traced by calorimetric measurements [16]. This glass transition of the IL was also observed previously by dynamic-mechanical analysis [14].

The $\alpha$ process at three temperatures is compared in Figure 2c. The addition of BMI to the CR rubber does not change the peak position and the broadness of the $\alpha$ process. Thus, the ionic liquid has no influence on the glass transition of the rubber matrix. This indicates that the two components are not very compatible and the solubility of the ionic liquid in the rubber matrix is low. In addition, the distinct presence of the $\alpha$ process of BMI is a hint that microdomains of BMI exist in the rubber matrix. Hence, BMI forms partly a separated phase that has its own glass transition at temperatures smaller than the glass transition temperature of the rubber matrix.

The large rise of dielectric loss at small frequency and large temperatures seen in both investigated samples is probably generated by ion conduction and electrode polarization, which become often dominant above the glass transition. Ionic conductance probably results from mobile ions obtained

Table 1. Curing recipe and explanation for sample designation. All units are in phr (per hundred rubber).

\begin{tabular}{|l|c|c|c|c|c|c|c|}
\hline Sample designation & CR rubber & ZnO & MgO & Stearic acid & Ethylene thiourea & MWCNTs & BMI \\
\hline CT0 & 100 & 5 & 4 & 0.5 & 1 & 0 \\
\hline CT0BMI20 & 100 & 5 & 4 & 0.5 & 1 & 0 \\
\hline CT3BMI0 & 100 & 5 & 4 & 0.5 & 1 & 3 \\
\hline CT3BMI15 & 100 & 5 & 4 & 0.5 & 1 & 3 \\
\hline
\end{tabular}



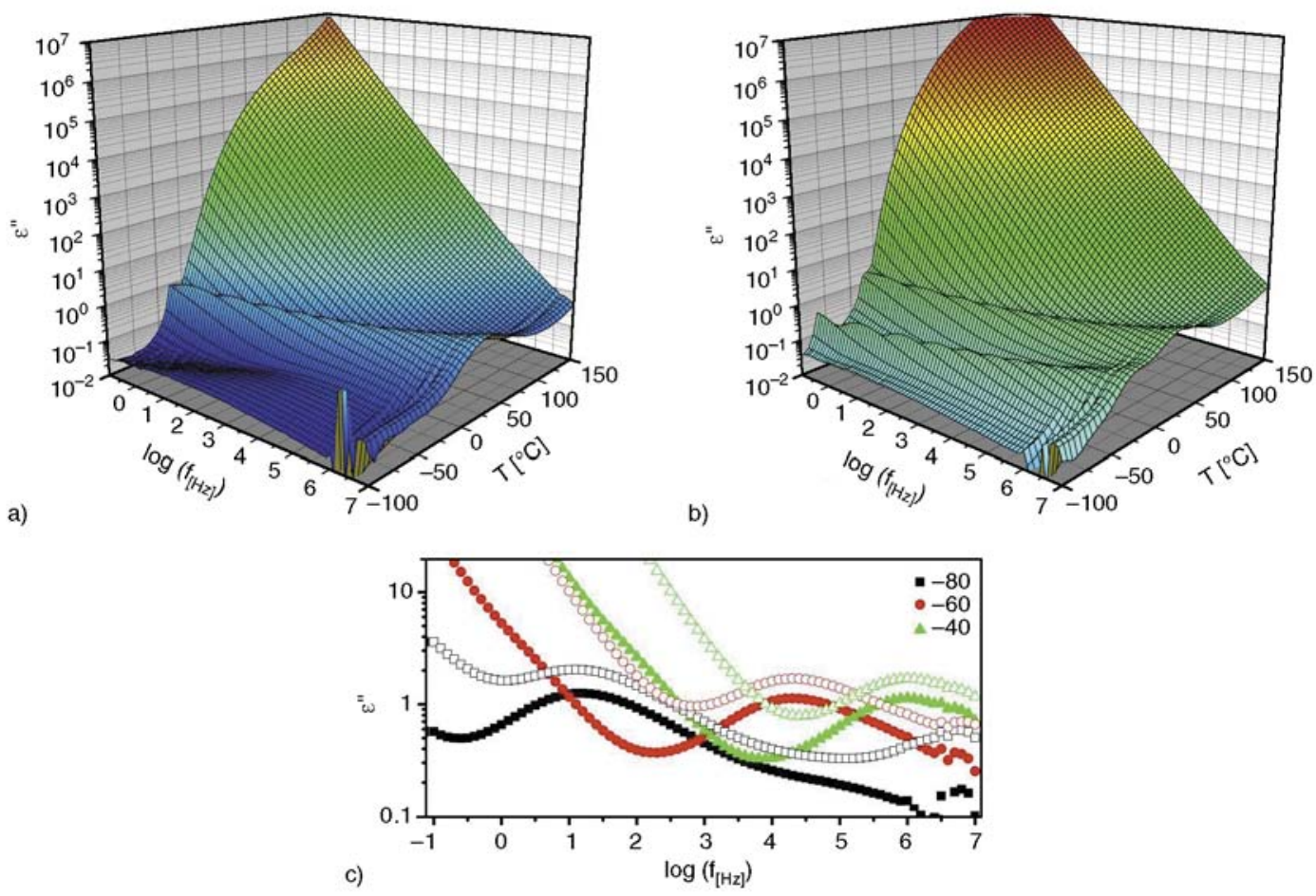

Figure 2. Dielectric loss of CR rubber a) without BMI (sample: CT0) and b) with BMI (sample: CT0BMI20). c) Comparison of the $\alpha$ process. Closed symbols: CT0; Open symbols: CT0BMI20.

from addition of the processing agents zinc oxide, magnesium oxide and stearic acid. It might be possible that this large effect masks relaxation transitions in the high temperature range. In order to minimize it, an analyzing procedure has been developed by Wübbenhorst and van Turnhout [17]. This method is based on the Kramer-Kronig relations, one of which is Equation (1):

$\varepsilon^{\prime \prime}=\frac{\sigma_{\mathrm{dc}}}{\varepsilon_{0} \omega_{0}}+\frac{2}{\pi} \int_{0}^{\infty} \varepsilon^{\prime}\left(\omega_{0}\right) \frac{\omega_{0}}{\omega^{2}-\omega_{0}^{2}} \mathrm{~d} \omega$

where $\sigma_{\mathrm{dc}}$ is the Ohmic conduction, $\omega_{0}$ is the relaxation frequency and $\varepsilon_{0}$ is the vacuum permittivity. It states that the real part $\varepsilon^{\prime}$ and the imaginary part $\varepsilon^{\prime \prime}$ are carrying the same information about relaxation processes. Other than the imaginary part $\varepsilon^{\prime \prime}$, the real part of permittivity $\varepsilon^{\prime}$ has no contribution from the frequency-independent Ohmic conduction $\sigma_{\mathrm{dc}}$. Therefore, determining the dielectric loss from the quantitiy $\varepsilon^{\prime}$ eliminates the part of conduction with ohmic behavior. In the analysis procedure of Wübbenhorst, the derivation of $\varepsilon^{\prime}$ with respect to the frequency $\omega$ denoted as $\varepsilon^{\prime \prime}{ }_{\text {deriv }}$ :

$$
\varepsilon_{\text {deriv }}^{\prime \prime} \approx-\frac{\pi}{2} \frac{\delta \varepsilon^{\prime}(\omega)}{\delta \ln \omega}
$$

is used in order to obtain narrow and sharp peaks and to eliminate ohmic conductivity. Equation (2) is almost exact and the derivative reproduces the measured frequency dependent $\varepsilon^{\prime \prime}$ data for broad relaxation processes such as the glass process. Contrary, for narrow Debye-like processes a peak sharpening is observed and $\varepsilon^{\prime \prime}{ }_{\text {deriv }} \propto \omega^{-2}$ is obtained for large frequencies instead of $\varepsilon^{\prime \prime}{ }_{\text {deriv }} \propto \omega^{-1}$ [17].

The partial blocking of ions at the metallic electrodes leads to the pronounced contribution in the measured $\varepsilon^{\prime \prime}$ at high temperature and low frequency. This electrode polarization refers typically to a Debye-like process with relaxation maximum below the measured frequency range. Therefore, Equation (2) is expected to sharpen this undesired peak and the contribution in the measured range is diminished, enabling the observation of other processes of dipolar origin.

The dielectric loss $\varepsilon^{\prime \prime}$ deriv evaluated by the derivative of $\varepsilon^{\prime}$ is shown for neat $\mathrm{CR}$ rubber in Figure 3a. An additional relaxation is clearly observed above the glass transition, which was masked in the measured dielectric loss $\varepsilon^{\prime \prime}$ in Figure 2a by the dominant effects related to ion conduction and electrode polarization. A comparison of $\varepsilon^{\prime \prime}$ deriv for the both investigated samples, CT0 without IL and CT0BMI20 

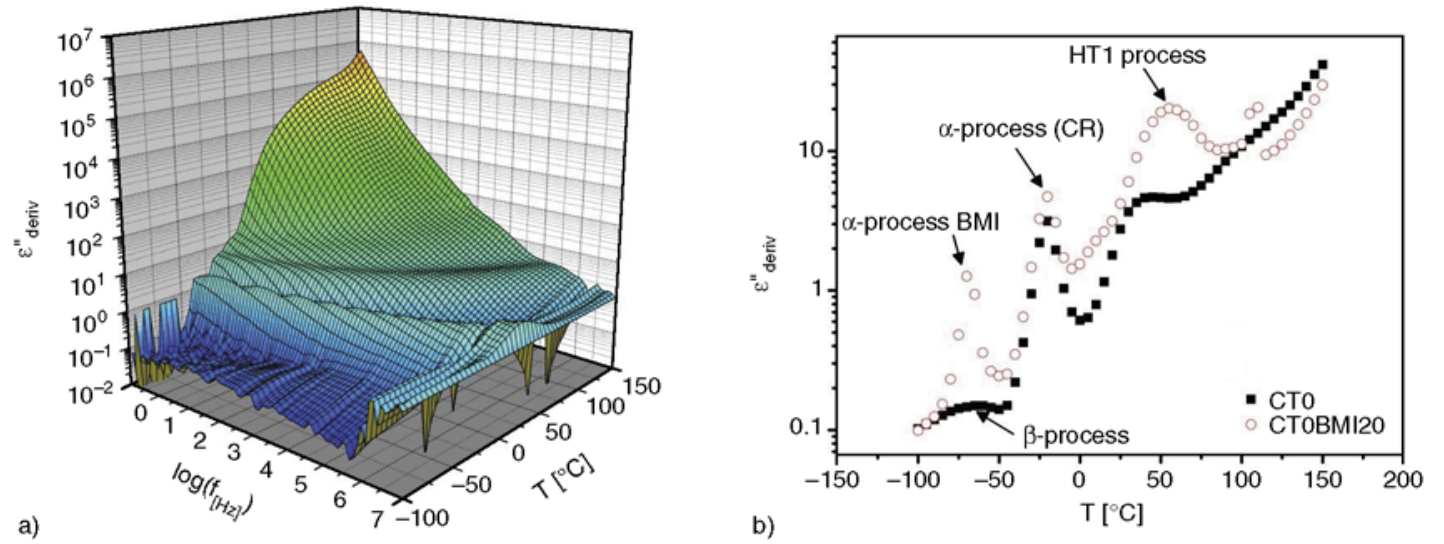

Figure 3. a) Dielectric loss from derivative of $\varepsilon^{\prime}$ for neat CR rubber (sample: CT0). b) Dielectric loss $\varepsilon^{\prime \prime}$ deriv for CT0 and CT0BMI20 at $f=1 \mathrm{kHz}$.

with IL, can be seen in Figure $3 \mathrm{~b}$ at $1 \mathrm{kHz}$. The glass transition of CR is clearly observed and is at the same temperature for both samples. Thus, the ionic liquid does not influence the glass transition of the rubber. The high temperature process, noted here as HT1, is obviously shifted to higher temperature for using BMI. This indicates that the ionic liquid hinders the relaxation belonging to this transition. At low temperatures, the $\beta$ process is observed for CT0 and the $\alpha$ process of BMI for CT0BMI20.

The dielectric spectra are fitted by Cole-Cole functions (Equation (3)):

$\varepsilon_{\mathrm{CC}}^{*}(\omega)=\varepsilon_{\infty}+\sum_{\mathrm{j}} \frac{\Delta \varepsilon_{\mathrm{j}}}{1+\left(i \omega \tau_{\mathrm{j}}\right)^{\alpha_{\mathrm{j}}}}$

where $\varepsilon_{\infty}$ is the dielectric permittivity at infinite frequencies, $\tau_{\mathrm{j}}$ is the relaxation frequency, $\Delta \varepsilon_{\mathrm{j}}$ is the relaxation strength and $\alpha_{\mathrm{j}}$ is the broadening parameter of the $j$-th relaxation process. The fit has been carried out for each temperature as shown in Figure 4 for $20^{\circ} \mathrm{C}$. At this temperature, the $\alpha$ process and the high temperature process HT1 are clearly visible.

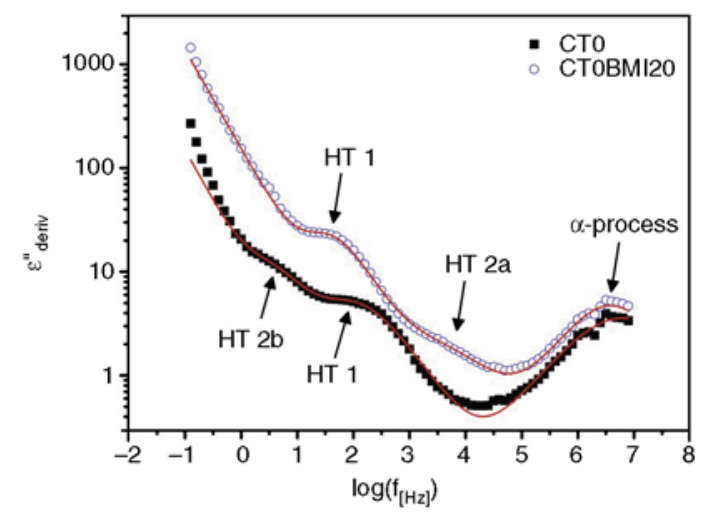

Figure 4. Fits of dielectric spectra with Cole-Cole functions at $20^{\circ} \mathrm{C}$. The lines are fits according to Equation (3).
Additionally, another high temperature process for each of the both samples, noted here as HT2a and HT2b, is observable. These processes are broad and have relatively small relaxation strengths. The determination is therefore difficult and imprecise. It is also unclear whether these both processes have the same origin or a different one.

The activation diagram, the logarithm of the relaxation frequency versus the reciprocal temperature, is shown in Figure 5. For each of the both samples, four processes have been detected. The $\alpha$ processes have been analyzed by the empirical Vogel-FulcherTamman equation (Equation (4)):

$f(T)=f_{0} \exp \left(\frac{-E_{\mathrm{A}}}{R\left(T-T_{\mathrm{FV}}\right)}\right)$

where $R$ is the gas constant. The activation energy $E_{\mathrm{A}}$, the Vogel-Fulcher temperature $T_{\mathrm{FV}}$ and the relaxation rate $\log f_{0}$ are the fit parameters. The results are summarized in Table 2 . It has been found that the $\alpha$ process of $\mathrm{CR}$ with the activation energy of about $10 \mathrm{~kJ} / \mathrm{mol}$ does not change with the usage

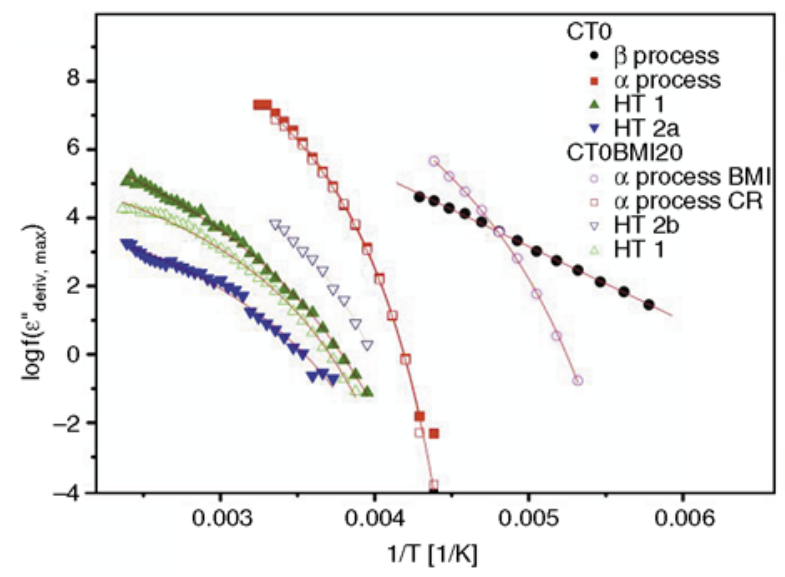

Figure 5. Activation diagram for the relaxation processes 
Table 2. Activation parameters for CT0 and CTOBMI20

\begin{tabular}{|l|c|c|c|}
\hline & $\begin{array}{c}\mathbf{E}_{\mathbf{a}} \\
{[\mathbf{k J} / \mathbf{m o l}]}\end{array}$ & $\log _{\mathbf{0}}$ & $\begin{array}{c}\mathbf{T}_{\mathbf{F V}} \\
{[\mathbf{K}]}\end{array}$ \\
\hline \multicolumn{4}{|c|}{ CT0 } \\
\hline$\beta$ process & 41.5 & 14 & - \\
\hline$\alpha$ process CR & 10 & 12.2 & 196 \\
\hline HT1 & 13.2 & 8.1 & 179 \\
\hline HT2a & 9.3 & 5.3 & 192 \\
\hline \multicolumn{4}{|c|}{ CT0BMI20 } \\
\hline$\alpha$ process CR & 9.7 & 11.9 & 196 \\
\hline$\alpha$ process BMI & 9.3 & 11.8 & 149 \\
\hline HT1 & 6.5 & 8.9 & 243 \\
\hline HT2b & 7.6 & 7.9 & 204 \\
\hline
\end{tabular}

of BMI. This indicates that the dynamics of the bulk polymer matrix is not affected by the presence of the ionic liquid. The $\alpha$ processes of the BMI can also well be fitted with the Vogel-Fulcher-Tamman equation. The activation energy and the relaxation rate are comparable to that of the CR polymer, but the Vogel-Fulcher temperature is much smaller. The $\beta$ process is well described by the Arrhenius relation (Eqiuation (5)):

$f(T)=f_{0} \exp \left(\frac{-E_{\mathrm{A}}}{R T}\right)$

having an activation energy of about $41.5 \mathrm{~kJ} / \mathrm{mol}$. The $\beta$ process is only observed in the neat rubber CT0. In CT0BMI20, the $\beta$ process is probably covered by the BMI $\alpha$ peak, which lies in the same temperature range.

It can be seen that all high temperature processes can well be described by a Vogel-Fulcher-Tammann behavior. The BMI clearly influences the high temperature process HT1. The relaxation rate is for both samples comparable, but the activation energy is smaller and the Vogel-Fulcher temperature is much larger for CT0BMI20 than for CT0. The origin of the relaxation process HT1 might be the formation of zinc clusters as recently reported in carboxylated nitril rubber (XNBR) [18]. In this study, it has been observed by dielectric relaxation spectroscopy and confirmed by IR spectroscopy that clusters are formed due to the presence of $\mathrm{ZnO}$ and carboxylic groups. In chloroprene rubber, a similar mechanism is imaginable between the polar side groups and the $\mathrm{ZnO}$. The high temperature process could therefore be assigned to the thermal decay of zinc clusters. The ionic nature of the IL can stabilize these clusters, explaining the shift to higher temperatures.

The used CR grade has a slight to medium tendency to crystallize [19]. Thus, a few crystalline regions might exist in the mostly amorphous polymer matrix. It is possible that the melting of these crystalline regions generates the less-pronounced, broad relaxation process HT2a in CT0. The melting temperature at long times of crystalline domains in chloroprene rubber is found at $30-70^{\circ} \mathrm{C}$ dependant on structural and geometrical isomerism [20] and lies therefore in the same temperature range as HT2a at low frequencies. This process is probably not observable in CTOBMI20 due to the higher electrode polarization and the shift of HT1 towards HT2a. HT2b in CT0BMI20 is maybe generated by the melting of BMI with melting point at $0^{\circ} \mathrm{C}$ that matches approximately HT2b at small frequencies.

\subsection{Dielectric properties of CNT filled rubber composites}

Figure 6 illustrates the dispersion of 3 phr CNTs in the CR rubber matrix by TEM pictures. Considering the sample CT3BMI0 (Figure 6a and 6c), the dispersion state of the tubes is poor and a large amount of different sized agglomerates are seen. The tube agglomerates are also spaced apart without intertubular connection in many regions. The pristine MWCNTs have a tendency to form entangled bundles, which are apparently hard to separate only by the shearing force during mixing with the elastomer matrix. In contrary, the TEM picture of CT3BMI15 (Figure 6b) shows only small agglomerates. At higher magnification (Figure 6d), single CNTs are seen, which are well distributed over the entire image. Thus, a much better dispersion can be obtained by modifying the CNTs with BMI. Additionally, the intertubular contacts are increased. It has been recently discussed that the Van der Waals attraction among the tubes decreases due to cation pi interaction between ILs and CNTs in modified tubes $[10,11]$. This leads to disentanglement of CNTs that in turn increased the dispersion and the formation of a highly conductive CNT network.

In Figure $7 \mathrm{a}$, the real part of conductivity is shown in dependence on temperature and frequency for the sample CT3BMI15, which contains 3 phr CNTs and 15 phr BMI. At small frequencies, a conductiv- 


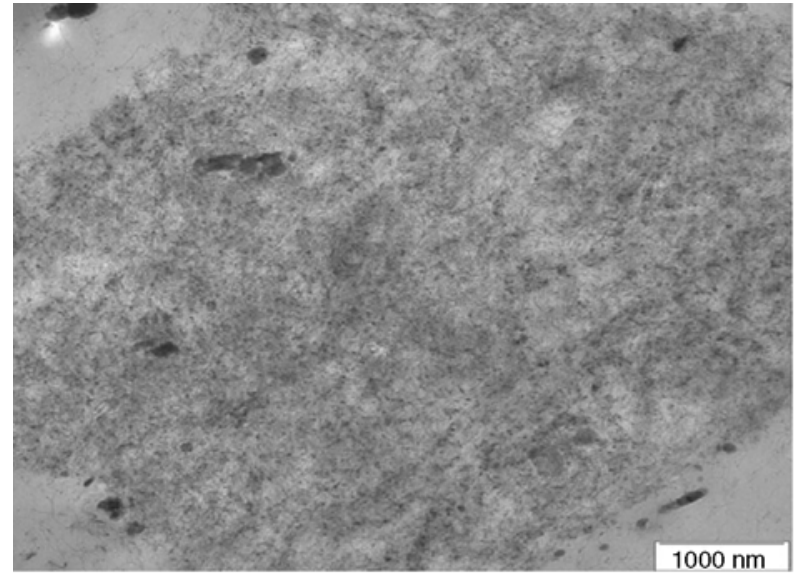

a)

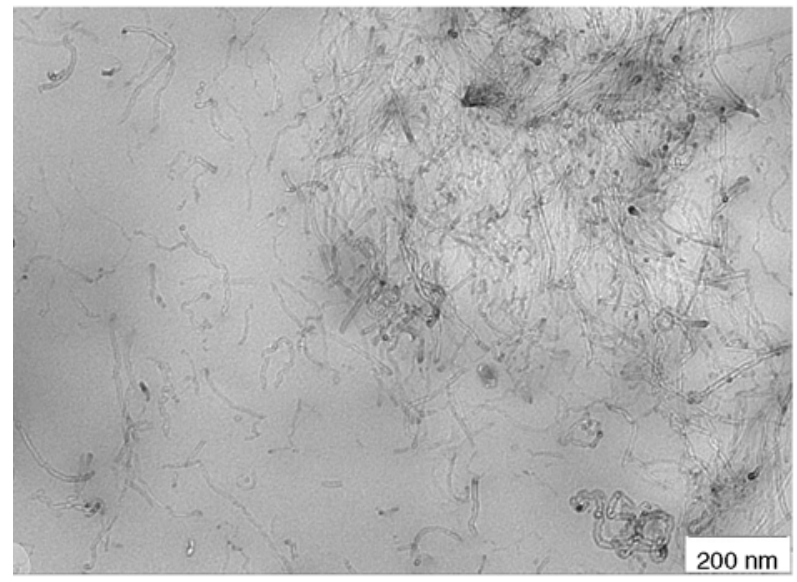

c)

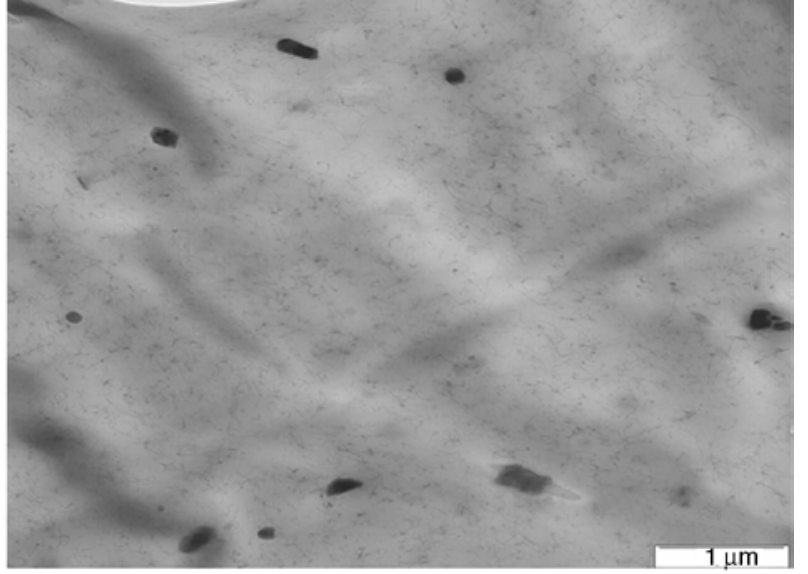

b)

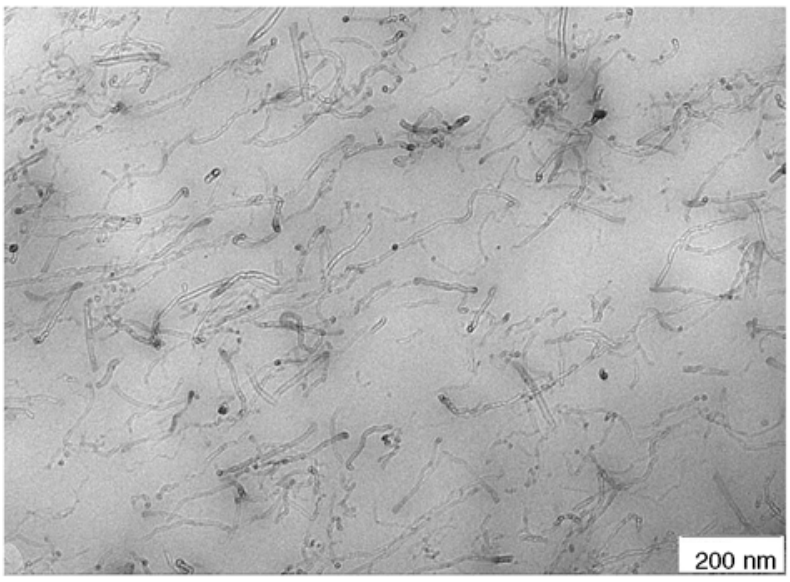

\section{.}

Figure 6. TEM images of CNT filled CR rubber. a) and c) CT3BMI0 without BMI. b) and d) CT3BMI15 with BMI. Scale bar: a), b) $1 \mu \mathrm{m}$ and c), d) $200 \mathrm{~nm}$.

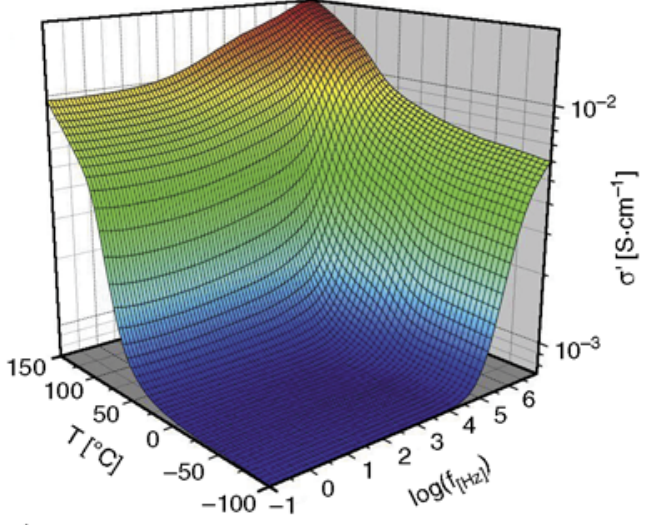

a)

Figure 7. a) Real part of the conductivity of CT3BMI15. CT3BMI15 (with IL).

ity plateau is observed which corresponds to the dc conductivity. The ac conductivity increases from the plateau into a disperse regime at about $10 \mathrm{kHz}$. The dc plateau is almost constant at small temperatures and increases strongly at around room temperature. The effect of ionic liquids on the dc conduc-

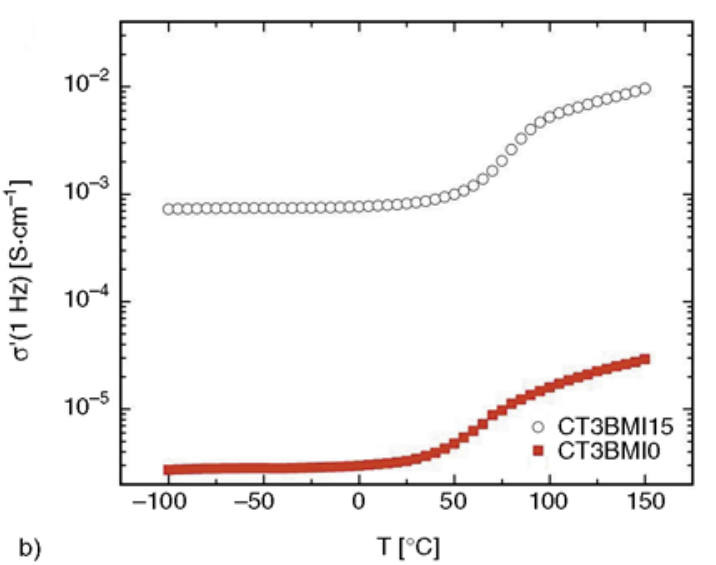

b) 
Neat CR rubber (sample CT0) and CR rubber containing BMI (CTOBMI20) have a conductivity at room temperature of $\sim 10^{-10} \mathrm{~S} / \mathrm{cm} \sim 10^{-9} \mathrm{~S} / \mathrm{cm}$, respectively. Thus, the ion conduction is insignificant compared to the much larger conductivity due to the electron transport through the CNT network.

One main reason for the much larger conductivity in the samples containing BMI is the improved dispersion. Indeed the conductivity is not only be determined by the structure and number of conductive paths of the CNT network, but also affected by a contact resistance between neighboring CNTs. As pointed out by Fritzsche et al. [13], quantum mechanical tunneling of electrons between neighboring CNTs plays a dominant role for CNT/rubber composites. CNTs in the rubber matrix have no direct geometrical contact, but are separated by a thin polymer layer that limits the dc conductivity of the CNT network. Despite these small gaps between the CNTs, electrons can overcome these barriers by tunneling or hopping. The increase in conductivity above room temperature suggests that a transition from tunneling to thermal activated hopping takes place and therefore the transport mechanism between CNTs changes. This behavior has also been observed for carbon black/rubber composites [6].

In order to gain more information about $\mathrm{CNT} / \mathrm{CNT}$ connections, the permittivity has been considered. The real part of permittivity at different temperatures can be seen in Figure 8. At small temperatures, a relaxation transition is found at relatively large frequencies. For CT3BMI15 the relaxation transition is sharper and at larger frequencies than for CT3BMI0. Additionally, the plateau of the permittivity at small frequencies has a much larger value for CT3BMI15, indicating a larger polarization in the modified

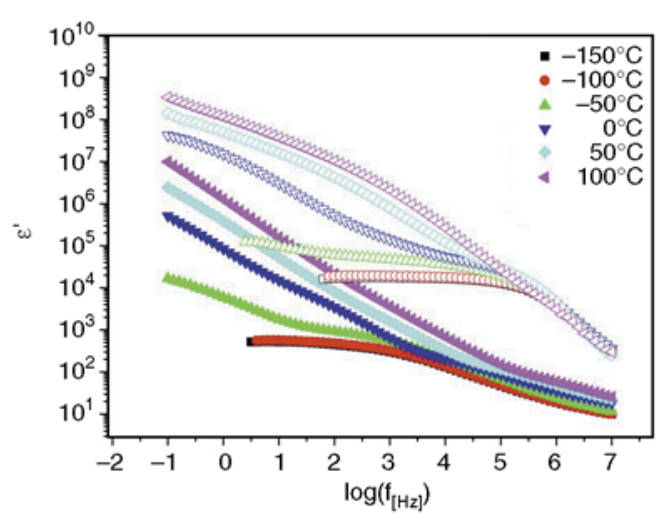

Figure 8. Real part of permittivity for CT3BMI0 (closed symbols) and CT3BMI15 (open symbols) at different temperatures
CNT/rubber composite. With increasing temperature, a large rise of permittivity at small frequencies becomes dominant. The high $\varepsilon^{\prime}$-values might be correlated to Maxwell-Wagner-Sillars relaxation that occurs due to accumulation of ions at the interfaces in inhomogeneous media. This effect becomes more important at larger temperatures since the thermal activated ion transport increases with increasing temperature.

The sharp relaxation transition at large frequencies can be traced back to a quantum mechanical tunneling process of electrons across small gaps between adjacent CNTs [13]. A relation between the relaxation frequency and the gap distance between neighboring filler particles has been derived, which says that larger relaxation frequencies means smaller gap distances. Consequently, the gaps between CNTs in the sample with IL (CT3BMI15) are smaller. Additionally, the sharper transition indicates a narrower distribution of gap distances. The much larger value of the relaxation strength for the IL modified CNT composite indicates that a larger amount of gaps exist which contribute to the polarization. This is consistent with the better dispersion and the more homogenous CNT network. The consequence of smaller gaps is that the quantum mechanical tunneling current over them is larger and thus the conductivity is increased. The main reasons for the improved conductivity of the IL modified CNT composite are therefore the smaller gaps between the CNTs and the better CNT networking.

The conductivity could additionally be influenced by the improved polymer-filler interaction due to BMI as coupling agent. The polymer in the nanoscopic gaps between neighboring fillers is expected to be glassy [6] because of immobilization of polymer chains by confinement between adjacent filler particles and adsorption of polymer segments at the surfaces. The stronger physical coupling by BMI probably affects the polymer dynamics in the gaps and therewith the dielectric properties. In order to estimate the influence of this effect on the conductivity, the measured permittivity of the $\mathrm{CR}$ without CNTs (CT0 and CTOBMI20) can be considered, which is for both samples around 4 in the glassy regime and around 10 in the elastic regime. Since the tunneling current is only proportional to the polymer permittivity [13], the influence on conductivity due to any changes in flexibility of the poly- 
mer is relatively small compared to the improved conductivity of more than two orders of magnitude. The effect of the gap size with an exponential dependence of gap distance on the tunneling current is probably much larger.

\section{Conclusions}

The dielectric spectra of unfilled chloroprene rubber and of IL/chloroprene rubber have been investigated. Besides electrode polarization, four relaxation processes have been detected for each of the both samples. It has been found that the glass transition described by a Vogel-Fulcher behavior does not change with the usage of ionic liquid, indicating that the both components are not compatible. The weakly pronounced, broad relaxation process at low temperature in pure $\mathrm{CR}$ rubber can be assigned to the $\beta$ process due to rotational motion of side groups. For the sample containing IL, the $\beta$ process is masked by an additional relaxation transition, which is much more pronounced. This process can be related to the $\alpha$ process of the IL, whose disordered and fragile structure typically exhibits a glassy like state at low temperatures. The origin of one of the both observed high temperature processes might be the existence of zinc clusters.

The high electrical conductivity of the CNT-filled rubber composites can be related to the transport of electrons along conductive paths of the throughgoing CNT network. The temperature behavior of the conductivity suggests that the conduction mechanism between neighboring CNTs is quantum mechanical tunneling at low temperatures and thermal activated hopping above room temperature. The ILmodification of the CNTs leads to an increased conductivity that is related to smaller gap distance between neighboring CNTs and a better dispersion due to a physical coupling between CNTs and rubber matrix.

\section{References}

[1] Chung D.: Materials for electromagentic interference shielding. Journal of Materials Engineering and Performance, 9, 350-354 (2000). DOI: $\underline{10.1361 / 105994900770346042}$
[2] Kost J., Narkis M., Foux A.: Resistivity behavior of carbon-black-filled silicone rubber in cyclic loading experiments. Journal of Applied Polymer Science, 29, 3937-3946 (1984).

DOI: 10.1002/app.1984.070291226

[3] Stübler N., Fritzsche J., Klüppel M.: Mechanical and electrical analysis of carbon black networking in elastomers under strain. Polymer Engineering and Science, 51, 1206-1212 (2011).

DOI: $10.1002 /$ pen.21888

[4] Chen L., Chen G. H., Lu L.: Piezoresistive behavior study on finger-sensing silicone rubber/graphite nanosheet nanocomposites. Advanced Functional Materials, 17, 898-904 (2007).

DOI: $10.1002 / \mathrm{adfm} .200600519$

[5] O'Farrell C. P., Gerspacher M., Nikiel L.: Carbon black dispersion by electrical measurements. Kautschuk Gummi Kunststoffe, 53, 701-710 (2000).

[6] Klüppel M.: The role of disorder in filler reinforcement of elastomers on various length scales. Advances in Polymer Science, 164, 1-86 (2003).

DOI: $10.1007 / \mathrm{b} 11054$

[7] Medalia A. I.: Electrical conduction in carbon black composites. Rubber Chemistry and Technology, 59, 432-454 (1986).

DOI: $10.5254 / 1.3538209$

[8] López-Manchado M. A., Biagiotti J., Valentini L., Kenny J. M.: Dynamic mechanical and Raman spectroscopy studies on interaction between single-walled carbon nanotubes and natural rubber. Journal of Applied Polymer Science, 92, 3394-3400 (2004).

DOI: $10.1002 /$ app.20358

[9] Gojny F. H., Wichmann M. H. G., Fiedler B., Kinloch I. A., Bauhofer W., Windle A. H., Schulte K.: Evaluation and identification of electrical and thermal conduction mechanisms in carbon nanotube/epoxy composites. Polymer, 47, 2036-2045 (2006).

DOI: $10.1016 /$ j.polymer.2006.01.029

[10] Das A., Stöckelhuber K. W., Jurk R., Fritzsche J., Klüppel M., Heinrich G.: Coupling activity of ionic liquids between diene elastomers and multi-walled carbon nanotubes. Carbon, 47, 3313-3321 (2009). DOI: $10.1016 /$ j.carbon.2009.07.052

[11] Lorenz H., Fritzsche J., Das A., Stöckelhuber K. W., Jurk R., Heinrich G., Klüppel M.: Advanced elastomer nano-composites based on CNT-hybrid filler systems. Composites Science and Technology, 69, 2135-2143 (2009).

DOI: 10.1016/j.compscitech.2009.05.014

[12] Shaffer M. S. P., Sandler J. K. W.: Carbon nanotube/ nanofibre polymer composites. in 'Processing and properties of nanocomposites' (ed.: Advani S. G.) World Scientific Publishing Company, Singapore (2006). 
[13] Fritzsche J., Lorenz H., Klüppel M.: CNT based elastomer-hybrid-nanocomposites with promising mechanical and electrical properties. Macromolecular Materials and Engineering, 294, 551-560 (2009).

DOI: $10.1002 /$ mame.200900131

[14] Subramaniam K., Das A., Heinrich G.: Development of conducting polychloroprene rubber using imidazolium based ionic liquid modified multi-walled carbon nanotubes. Composites Science and Technology, 71, 1441-1449 (2011).

DOI: 10.1016/j.compscitech.2011.05.018

[15] Subramaniam K., Das A., Steinhauser D., Klüppel M., Heinrich G.: Effect of ionic liquid on dielectric, mechanical and dynamic mechanical properties of multi-walled carbon nanotubes/polychloroprene rubber composites. European Polymer Journal, 47, 2234 2243 (2011).

DOI: 10.1016/j.eurpolymj.2011.09.021
[16] Yamamuro O., Minamimoto Y., Inamura Y., Hayashi S., Hamaguchi H.: Heat capacity and glass transition of an ionic liquid 1-butyl-3-methylimidazolium chloride. Chemical Physics Letters, 423, 371-375 (2006). DOI: $10.1016 /$ j.cplett.2006.03.074

[17] Wübbenhorst M., van Turnhout J.: Conduction-free dielectric loss $\partial \varepsilon / \partial \ln f-$ A powerful tool for the analysis of strong (ion) conducting dielectric materials. Dielectrics Newsletter, 14, 1-3 (2000).

[18] Fritzsche J., Das A., Jurk R., Stöckelhuber K. W., Heinrich G., Klüppel M.: Relaxation dynamics of carboxylated nitrile rubber filled with organomodified nanoclay. Express Polymer Letters, 2, 373-381 (2008). DOI: 10.3144 /expresspolymlett.2008.44

[19] Kempermann T.: Handbuch für die Gummiindustrie. Bayer AG, Leverkusen (1991).

[20] Batzer H.: Polymere Werkstoffe, Band III. Georg Thieme Verlag, New York (1984). 